\title{
Study on Dynamic Simulation of Cam Mechanism Based on Prol E and ADAMS
}

\author{
Yuekan Zhang ${ }^{1, a}$, Ruichuan $\mathrm{Li}^{2, \mathrm{~b}}$, Linjing Xiao ${ }^{1, \mathrm{c}}$, JunruYang ${ }^{1, \mathrm{~d}}$ \\ ${ }^{1}$ College of Mechanical \& Electronic Engineering SDUST, Qingdao, 266590 China \\ ${ }^{2}$ Shandong Wuzheng Group Co., Ltd. Rizhao, 262306 China

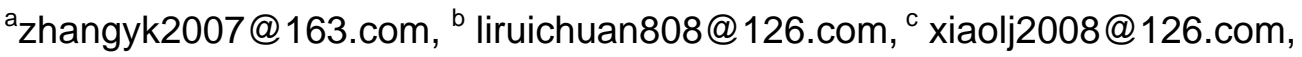 \\ d jryangzhang@163.com
}

Keywords: Cam mechanism, Dynamic simulation, Pro/E, Adams

Abstract. The virtual prototype system framework of the cam mechanism is established. The three-dimension (3-D) digital model of the cam is built with Pro/E. Dynamic simulation analysis of the cam mechanism is studied by means of software ADAMS. The results supply the bases for the structure optimization of the cam mechanism.

\section{Introduction}

Virtual prototype technology is a technique which establishes digital virtual prototype model consistent with the physical prototype, and uses the model to make multidisciplinary analyses [1]. Virtual prototype technology has become an important means to develop the complex products. It provides a new mode to convert product development process from "design-prototype manufacture-test analysis" to "modeling-digital simulation-product prototype". In the paper, the three-dimension (3-D) digital model of the cam is built with Pro/E [2] and dynamic simulation analysis of the cam mechanism is studied by means of software ADAMS[3].

\section{3-D Modeling of the Cam mechanism Virtual Prototype}

Taking roller cam mechanism as the object, according to the actual structure of the roller cam, utilizing Pro/E software, 3-D models of the roller cam parts are structured. The finished part models are assembled according to the design requirement. The roller cam assembly model can be constituted by multiple subassemblies such as the cam, the roller, the push rod, the guideway and return spring. Fig. 1 shows the assembled cam mechanism model.

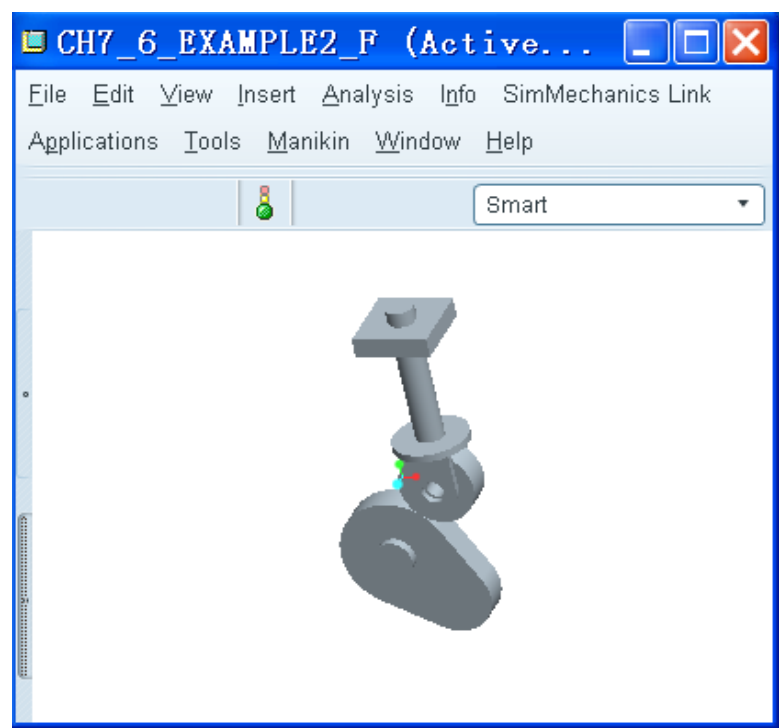

Fig.1 The assembled cam mechanism model

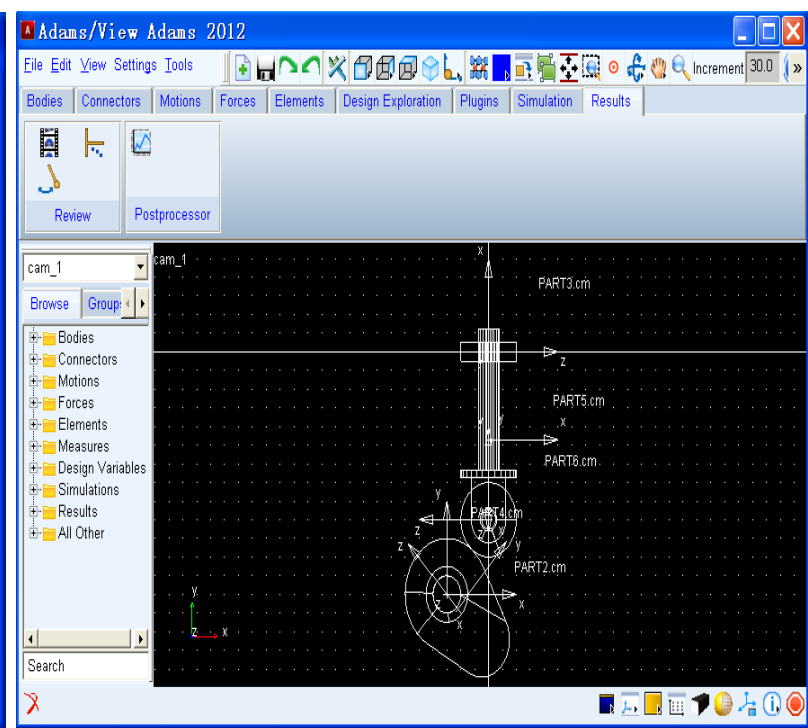

Fig. 2 The cam model in ADAMS 


\section{Dynamic Simulation of Cam Mechanism}

To load model into Adams, we have to put it into a format that will be understood. Therefore, the file in the Pro/E should be saved as the XT format. Then we open the software Adams, and click "file" $\longrightarrow$ "import", in the file type dialog box, we should select "parasolid". The cam mechanism model in the Pro/E is put into Adams. Fig.2 shows the cam model in Adams.

Revolute joint (Two bodies-One Location, Normal To Grid) between the cam and the ground is built, Revolute joint (Two bodies-One Location, Normal To Grid) between the roller and the push rod is built, translational joint(Two bodies-One Location, direction aligned geometry feature) between the push rod and the guideway is built, fixed joint (Two bodies-One Location, Normal To Grid) between the guideway and the ground is built, contact force between the cam and the roller is created, spring damping between the roller and the push rod is created, then rotational motion is applied to the cam rotational joint. The simulation model is shown as Fig.3.

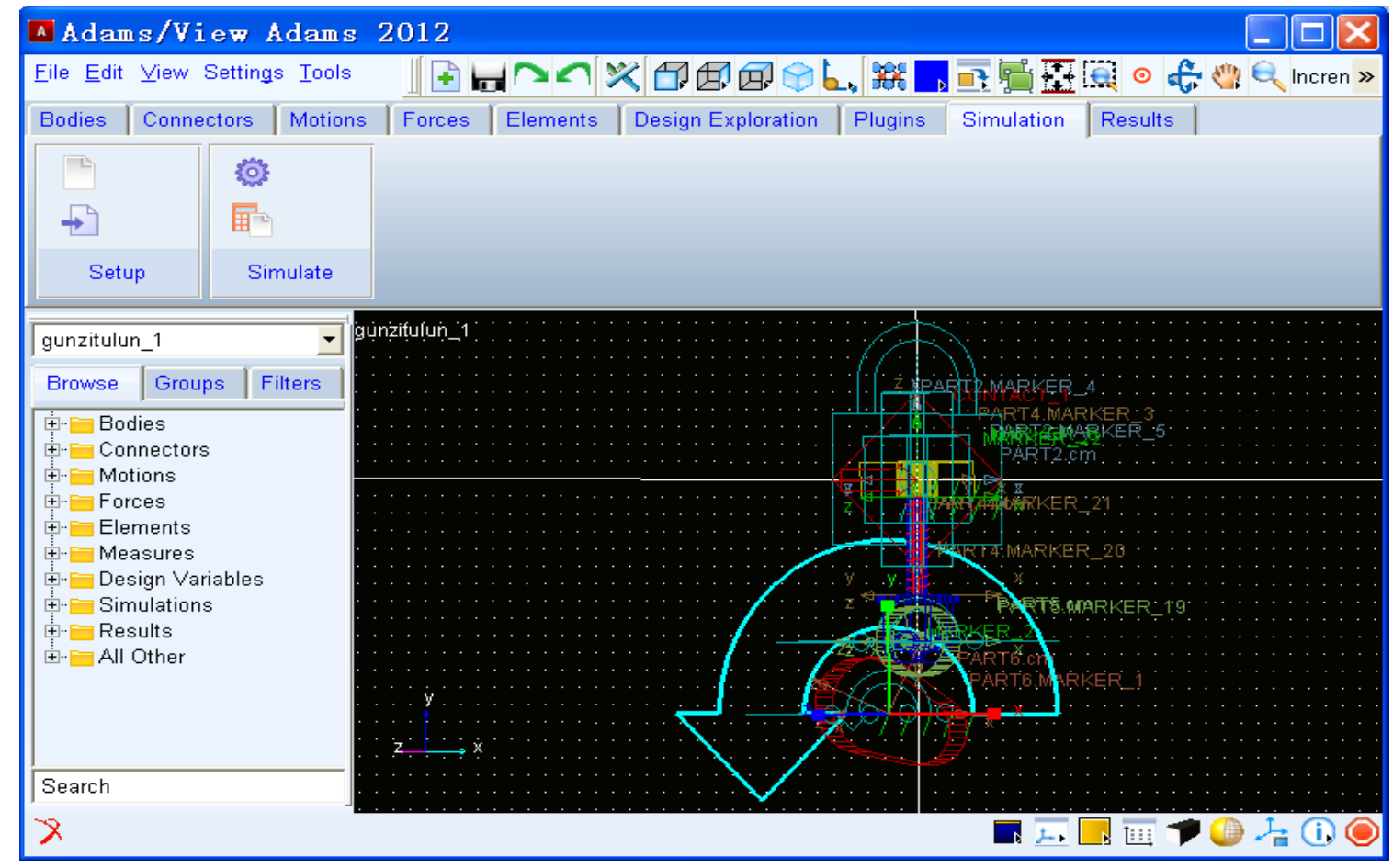

Fig.3The simulation modele

The rotational speed is 60 , simulation end time is $12 \mathrm{~s}$ and simulation steps are 300 , then the cam mechanism simulation is started. During the process of the cam simulation, we can observe the real-time visualization, which can display high quality realistic picture was executed under the simulation and the result can be received as the same as real motioning. The simulation results are finally extracted after post-proposing[4,5], the results are shown in Fig.4-Fig.8, Fig.4 shows the cam follower displacement curve, Fig.5 shows the cam follower velocity curve and Fig. 6 shows the cam follower acceleration curve. In the paper, Contact force[6] is an essential issue of simulation systems which intends to have more realistic motion of the cam mechanism in virtual environments. Fig. 5 and Fig. 6 show that under the contact force the cam follower speed and acceleration change frequently and largely. The results show that the simulation results are consistent with actual cam mechanism situation. After the filtering process, the cam follower velocity and acceleration curves are obtained, which are shown in Fig.7 and Fig.8. Fig.9 shows contact force between the cam and the push rod and spring force, The results supply the bases for the structure optimization of the cam mechanism. 


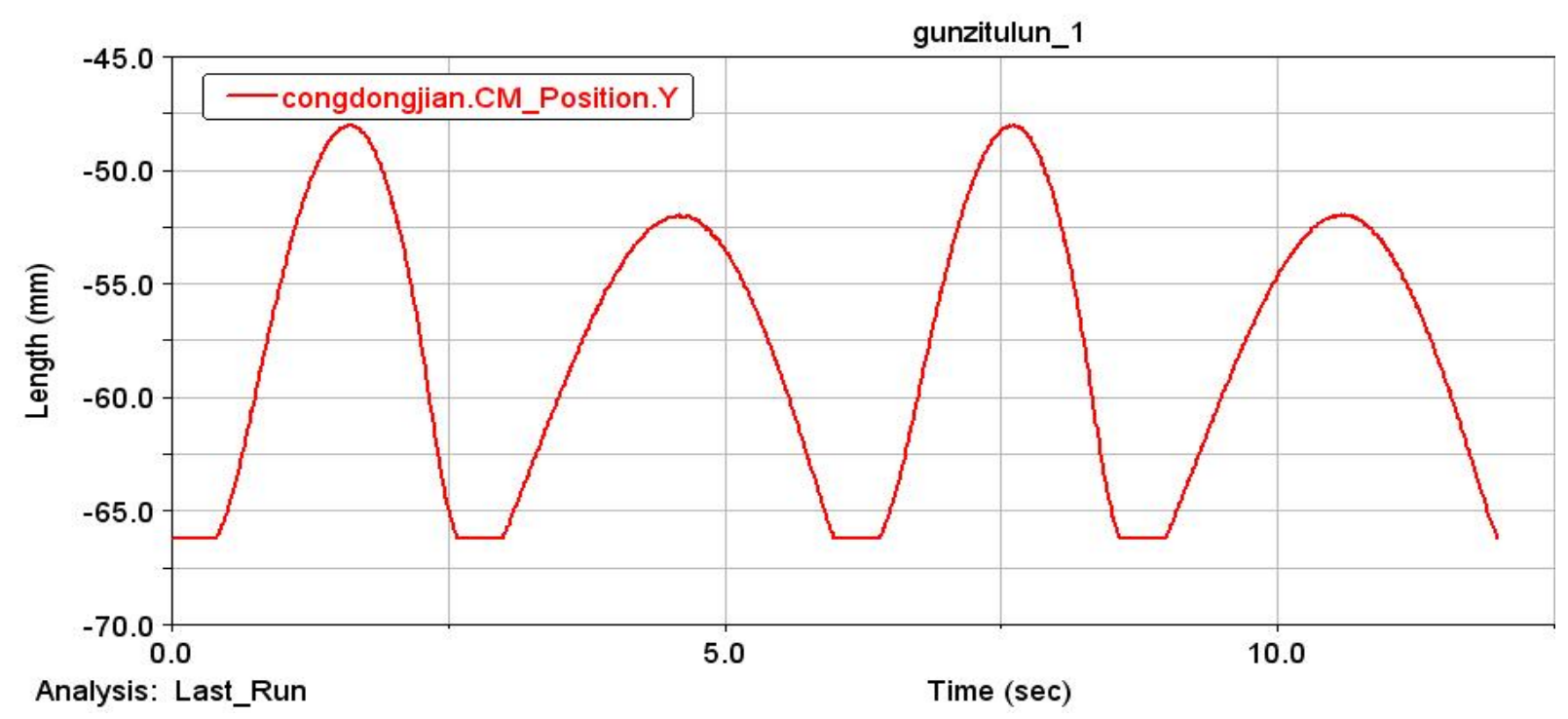

Fig.4 Cam follower displacement curve

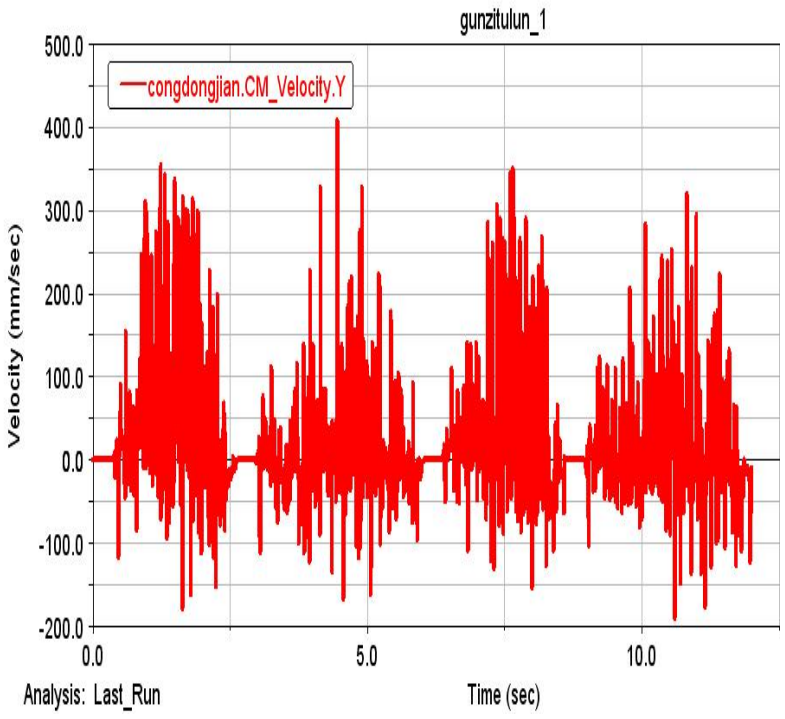

Fig.5 The cam follower velocity curve

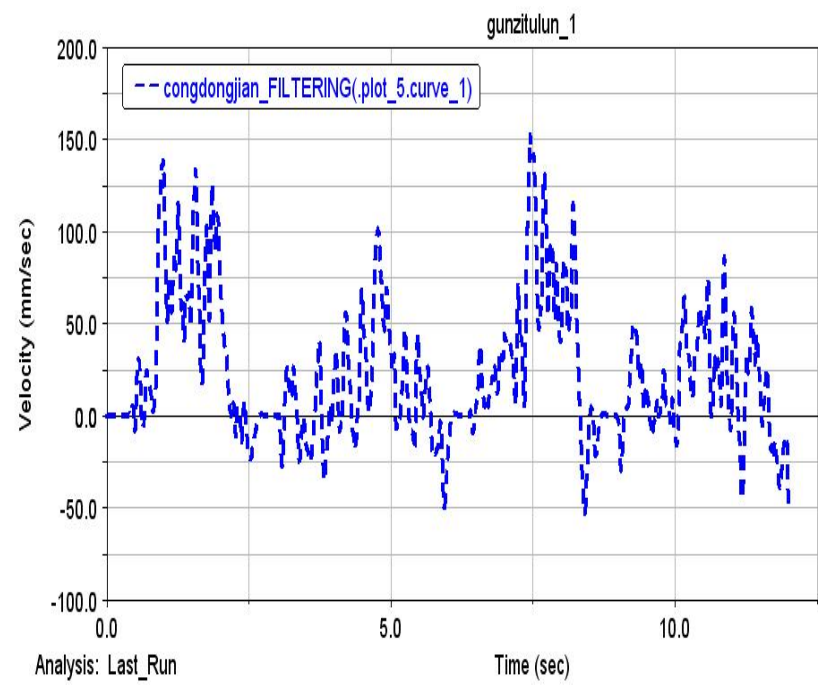

Fig7.After filtering the cam follower velocity curve

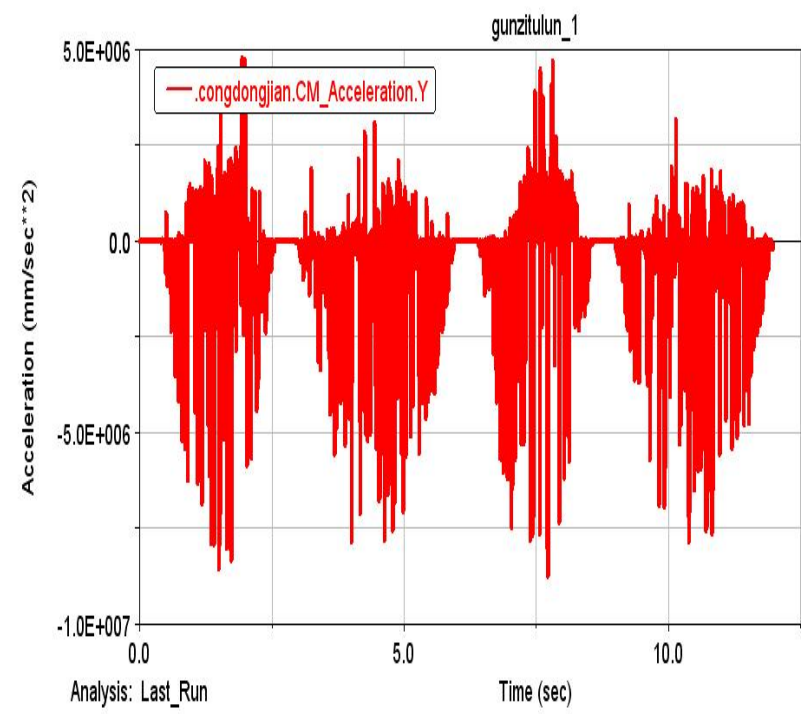

Fig.6 The cam follower acceleration curve

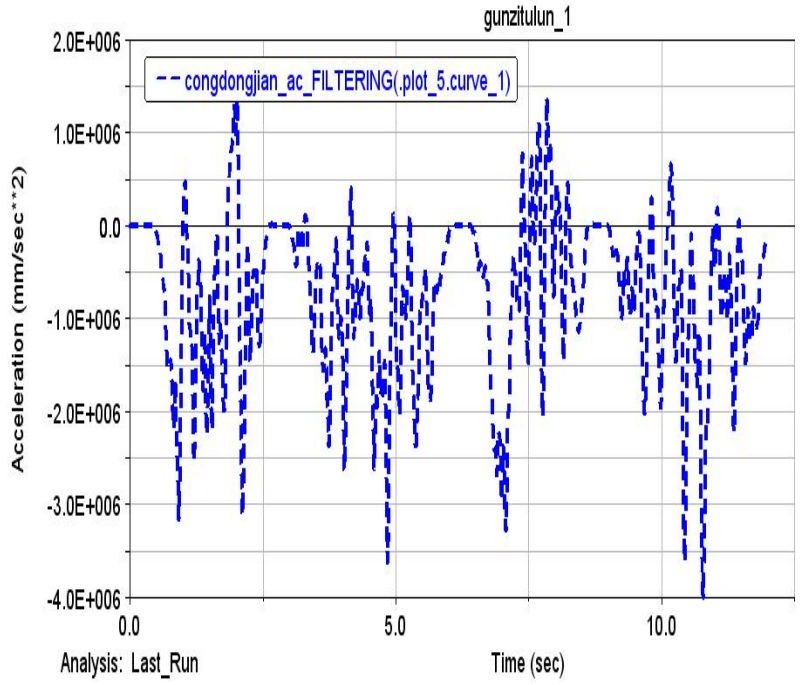

Fig8.After filtering the cam follower acceleration curve 


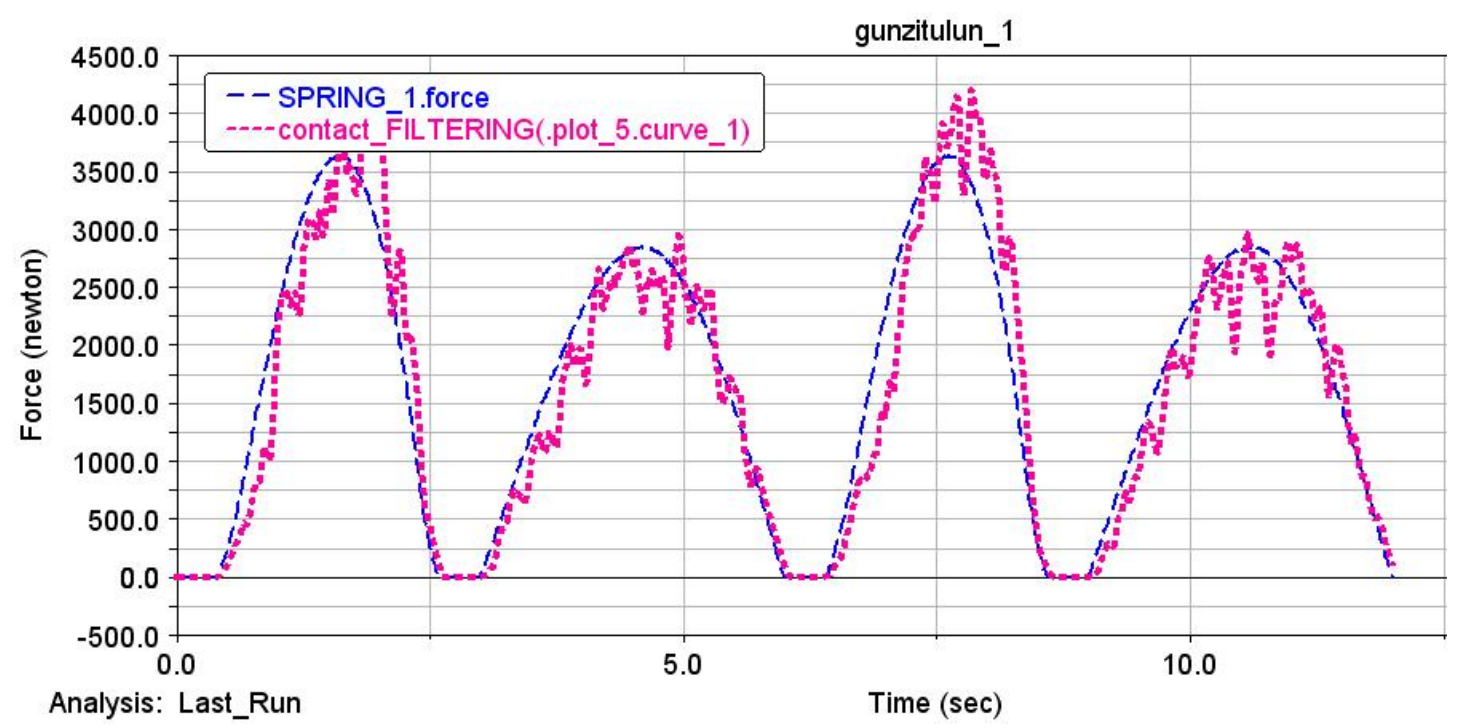

Fig.9 The curve of contact force and spring force

\section{Conclusions}

In the paper, based on the virtual prototype technology, with the interface between the software Pro/E and Adams, the model in Pro/E can easily transmitted to Adams, Data transmission is realized between Pro/E and Adams, which is helpful for Pro/E and Adams to develop their speciality and their associated simulation, the results are of great reference value for the future research. Main conclusions are obtained as the following.

(1) The virtual prototype system framework of the cam mechanism is established. The three-dimension (3-D) digital model of the cam is built with Pro/E.

(2) Dynamic simulation analysis of the cam mechanism is studied by means of software ADAMS. The data of cam follower's displacement, velocity and acceleration are obtained.

\section{Acknowledgements}

This work is supported by science and technology develop project of Shandong Province (2011GGA07130) and national high technology research and development program of China ( 863 Program, 2012AA040903).

\section{References}

[1] Guangleng Xiong, Bin Guo and Xixaobo Chen: Collaborative Simulation and Virtual Prototype Technology (Tsinghua University Press, China 2004).

[2] Shuhui Ding:Advanced design and practice with Pro/Engineer Wildfire 5.0(Tsinghua University Press, China 2010).

[3] Zenggang Li: elementary course and examples with ADAMS. National Defense Industry Press, China 2010).

[4] Qin Cheng:Mechanical Engineering \& Automation.No.4(2008),p35.

[5] Fei Liu, Dawei Zhang: Mechanical research \& Application.No.1(2011),p60.

[6] http://wenku.baidu.com/view/f12e28ef4afe04a1b071de63.html. 